\title{
MicroRNA-217 Functions as a Tumour Suppressor Gene and Correlates with Cell Resistance to Cisplatin in Lung Cancer
}

\author{
Junhua Guo*, Zhijun Feng, Zhi'ang Huang, Hongyan Wang, and Wujie Lu
}

\begin{abstract}
MiR-217 can function as an oncogene or a tumour suppressor gene depending on cell type. However, the function of miR-217 in lung cancer remains unclear to date. This study aims to evaluate the function of miR-217 in lung cancer and investigate its effect on the sensitivity of lung cancer cells to cisplatin. The expression of miR-217 was detected in 100 patients by real-time PCR. The effects of miR-217 overexpression on the proliferation, apoptosis, migration and invasion of SPC-A-1 and A549 cells were investigated. The target gene of miR-217 was predicted by Targetscan online software, screened by dual luciferase reporter gene assay and demonstrated by Western blot. Finally, the effects of miR-217 up-regulation on the sensitivity of A549 cells to cisplatin were determined. The expression of $\mathrm{miR-217}$ was significantly lower in lung cancer tissues than in noncancerous tissues $(p<0.001)$. The overexpression of miR-217 significantly inhibited the proliferation, migration and invasion as well as promoted the apoptosis of lung cancer cells by targeting KRAS. The up-regulation of miR-217 enhanced the sensitivity of SPC-A-1 and A549 cells to cisplatin. In conclusion, miR-217 suppresses tumour development in lung cancer by targeting KRAS and enhances cell sensitivity to cisplatin. Our results encourage researchers to use cisplatin in combination with miR-217 to treat lung cancer. This regime might lead to low-dose cisplatin application and cisplatin side-effect reduction.
\end{abstract}

\section{INTRODUCTION}

Lung cancer is the leading cause of cancer-related mortality worldwide (Jemal et al., 2010). Lung cancer has been traditionally subdividedinto two principal groups, namely, neuroendocrine and non-small cell lungcancer (NSCLC). The latter type is more common than the former. Cancer occurs and develops as a

Department of Respiratory, The First Hospital Affiliated to Henan University, Henan 475000, China

*Correspondence: junhuaguo2008@hotmail.com

Received 12 May, 2014; revised 20 July, 2014; accepted 31 July, 2014 published online 18 September, 2014

Keywords: cisplatin, KRAS, lung cancer, miR-217, tumour suppressor complicated result of an accumulation of various endogenous and exogenous effects. Gene alterations participate in cancer genesis. Alterations in many oncogenes and tumour suppressor genes have been reported in lung cancer.

MicroRNAs (miRNA) are a class of small RNA molecules that regulate the translation and degradation of mRNAs (Tomari and Zamore, 2005). MiRNAs bind to complementary sequences in the 3'-untranslated regions (UTRs) of their target mRNAs to promote $\mathrm{mRNA}$ degradation or translational repression (Engels and Hutvagner, 2006). MiRNAs function in negative gene regulation. These molecules silence gene expression by interfering with mRNA stability or protein translation.

MiRNAs participate in numerous biological processes, such as proliferation, apoptosis, differentiation and invasion. During tumourigenesis, miRNAs act as an oncogene or a tumour suppressor gene and contribute to cancer initiation and progression by regulating target genes (Ambs et al., 2008). MiRNAs function in tumourigenesis and metastasis by directly targeting oncogenes or tumour suppressor genes (Garzon et al., 2009; Slack and Weidhaas, 2008). MiRNAs such as miR-17-92 cluster (Inomata et al., 2009), miR-21 (Qi et al., 2009) and miR-31 (Zhang et al., 2011) function as oncogenes. Conversely, miRNAs such as miR-451 (Wang et al., 2011), miR-15/16 (Cimmino et al., 2005), let-7 (Johnson et al., 2005), miR125a/125b (lorio et al., 2007) and miR-145 (Schepeler et al., 2008) function as tumour suppressor genes. Meanwhile, some miRNAs can function as oncogenes or tumour suppressor genes depending on cell type. For example, miR-96 decreases FOXO1 protein levels and contributes to the survival of breast cancer cells (Guttilla and White, 2009). Alternatively, miR-96 induces tumour suppression in pancreatic cancer cells by targeting KRAS (Yuet al., 2010a). Similarly, miR-217 targets the oncogene SirT1 or KRAS in endothelial and pancreatic ductal adenocarcinoma cells (Menghini et al., 2009; Zhao et al., 2010) but targets the tumour suppressor gene PTEN (Kato et al., 2009). However, the function of miR-217 in lung cancer remains unclear to date.

The relationship between miRNAdysregulation and human cancer resistance has attracted increasing attention (Ma et al., 2010). MiRNAdysregulation can contribute to cisplatinchemoresistance in human tumour cells (Sorrentino et al., 2008; Yu et al., 2010b). In NSCLC, miRNA-451 can up-regulate cisplatin sensitivity (Bian et al., 2011). However, the association of other miRNA expression with the sensitivity of lung cancer cells to 
cisplatin has yet to be explored. The present study aims to provide insights into the association of miR-217 expression with cisplatin cell resistance in lung cancer. Results show that miR217 is down-regulated in human lung cancer and that miR-217 overexpression can reduce cisplatin cell resistance in lung cancer cell lines. Our in vitro and in vivo data also prove that miR217 functions as a tumour suppressor in human lung cancer progression. The results of this study may serve as a basis to explain the function of miR-217 in cisplatinchemoresistance and discover novel targeted combinations agents against lung cancer.

\section{MATERIALS AND METHODS}

\section{Patients and tissue samples}

We collected pairs of matched lung cancer and noncancerous tissue samples from 100 patients (male 56, female 44) who underwent surgical resection at the Cancer Institute and Hospital of Hebei; the Chongmin Hospital of Nanjing; First affiliated hospital of Henan University; Hebei Cancer Hospital between 2009 and 2012 after obtaining informed consent from all patients and receiving approval from the Institutional Ethics Review Committee. The lung cancer cell lines SPC-A-1 and A549 were cultured in RPMI 1640 or Dulbecco's modified Eagle's medium, with $10 \%$ FBS, $100 \mathrm{U} / \mathrm{ml}$ penicillin as well as streptomycin.

RNA extraction, cDNA synthesis, and real-time PCR assays Total RNA from tissues and cells was extracted byTrizolmethod (Ambion, USA) completely following the instructions. Single strand cDNA was synthesized by M-MLV (Ambion, USA) using 2 $\mu \mathrm{g}$ of total RNA as template. Oligo (dT) 18 was used for mRNA reverse transcription while stem loop used for miRNA. Real time-PCR (RT-PCR) was performed by Bio-rad CFX96 (Bio-rad, USA) using SYBR mix (Tiangen, China). The PCR condition was: $95^{\circ} \mathrm{C} \times 30 \mathrm{~s}$, followed by 40 cycles of $95^{\circ} \mathrm{C} \times 5 \mathrm{~s}, 60^{\circ} \mathrm{C} \times 34$ s. For mRNAs, GAPDH was used as normalized control. For miRNAs, U6 snRNA was used for miRNA control. The relative expression of miR-217 was computed by $2^{-\Delta \Lambda \mathrm{CT}}$ method. Primer sequences have been shown in Supplementary Table 1.

\section{Plasmid construction}

The miR-217 precursor sequence was generated by annealing MiR-217-precursor-F and miR-217-precursor-R extension was digested by $\mathrm{BamHI}$ and Bglll. The products were then inserted into the BamHI-Bglll fragment of the pcDNA-GW/EmGFP-miR vector (Gene Pharma, China). A negative control was also constructed.

\section{MiRNA transfection}

The miR-217 and scramble mimics were designed and synthesised by GenePharma (GenePharma, China). All mimics were transfected by DharmaFECT1 Reagent (Dharmacon, USA) into the lung cancer cells to a final concentration of $10 \mathrm{nM}$. Three independent replication experiments were performed for each transfection.

\section{Cell proliferation and apoptosis assay}

Cell proliferation assay was performed by the CCK8 method (DOJINDO, Japan). Briefly, approximately 5000 miR-217 mimictransfected cells and scramble cells were seeded into 96-well plates and then cultured. Proliferation rates were determined at $0,12,24,48,72$ and $96 \mathrm{~h}$ after transfection by adding $10 \mu \mathrm{l}$ of CCK8 reagent.

Apoptosis assays were tested in SPC-A-1and A549 cell lines with or without miR-217 overexpression using Apoptosis Detec- tion kit I (BD Biosciences, USA) and C6 Flow Cytometer (USA).

\section{Cell migration and invasion assay}

We performed the wound-healing assay to test cell migration ability with or without miR-217 mimic transfection. The artificial wounds wereproduced on the confluent cell monolayer with FBS free, using a $200 \mu \mathrm{l}$ pipette tip at $24 \mathrm{~h}$ post miR-217 transfection. The images were respectivelytaken at $0,12,24$ and $36 \mathrm{~h}$ after wound creation.

We then performed transwell assays to evaluate cells'invasionability. The $5 \times 10^{4}$ cells suspended in $200 \mu \mathrm{l}$ medium without FBS were placed on the upper chamber of each insert with $430 \mu \mathrm{l}$ of 1 $\mathrm{mg} / \mathrm{ml}$ matrigel(Millipore, USA). The $600 \mu \mathrm{l}$ medium with $10 \%$ FBS as the nutritional attractant was put in the lower chamber. After $24 \mathrm{~h}$, the cells attached to the lower surface of chamber were fixed 20 min by $20 \%$ methanol and stained for 10 min with $10 \%$ maygruwald-giemsa (MGG). The invasion membranes were then cut down and embedded under cover slips. We counted the cells in 3 different vision fields in condition with 20x magnification which were then used as the average number of cells. All assays were performed in three independent experiments.

\section{Animal experiment}

Experiments involving animals were performed according to the Guide for the Care and Use of Laboratory Animals and the institutional ethical guidelines for animal experiments. Scrambletransfected and miR-217-overexpressing A549 cells $\left(6 \times 10^{6}\right.$ cells) were inoculated s.c. into the dorsal flanks of BALB/c nude mice (female, Nu/Nu, six week old). The mice were purchased from the Animal Centre of Henan University and raised under pathogen-free conditions. The tumour volume was measured for 5 weeks. All mice were killed, s.c. tumours were resected, and tumour weights were recorded.

\section{Immunohistochemistry}

Mouse tumour tissues were embedded into paraffin sections, treated for $2 \mathrm{~h}$ at $65^{\circ} \mathrm{C}$ and then deparaffinised. Before applying the primary antibodies at $4^{\circ} \mathrm{C}$ overnight, we carried out the antigen retrieval step. The slides were incubated with a secondary antibody for $2 \mathrm{~h}$ at $25^{\circ} \mathrm{C}$ and then conjugated to HRP (1:100; Zhongshanjinqiao, China). Liquid DAB+ Substrate (zsgb-bio, China) was used to detect HRP activity.

Luciferase miRNA target reporter assay

The full length of the 3'UTRs of 6 human genes, including KRAS MAPK1, ACVR2A, TPD52L2, NOVA1, MAF, who have complementary sequences in their 3'UTR predicting by Targetscan on line software, were amplified by PCR and inserted into the pMIR-REPORTTM Luciferase Reporter Vector (Ambion, USA). Mutations of the predicted seed regions in the mRNA sequences were created using primers including the mutated sites. HEK293T cells were transfected with $\mathrm{pRL}-\mathrm{TK}$ luciferase reporters (50 ng/well, 24-well plates, $1 \times 10^{5}$ cells per well), pGL-3firefly luciferase (10 ng/well), and mimic-217 (50 nmol/L, GenePharma, China) or scramble (50 nmol/L) using Lipofectamine 2000 (Invitrogen, USA). Luciferase activities were measured according to the manuscript of the Dual Luciferase Reporter Assay (Promega, USA).

\section{Western blot}

Proteins were separated on $10 \%$ SDS-PAGE and then transferred to $0.45 \mu \mathrm{m}$ PVDF membranes (Amersham, UK). The membranes were incubated with $5 \%$ non-fat dried milk overnight at $4^{\circ} \mathrm{C}$ and with anti-KRAS antibody (CST, USA) at 1:1000 dilu- 

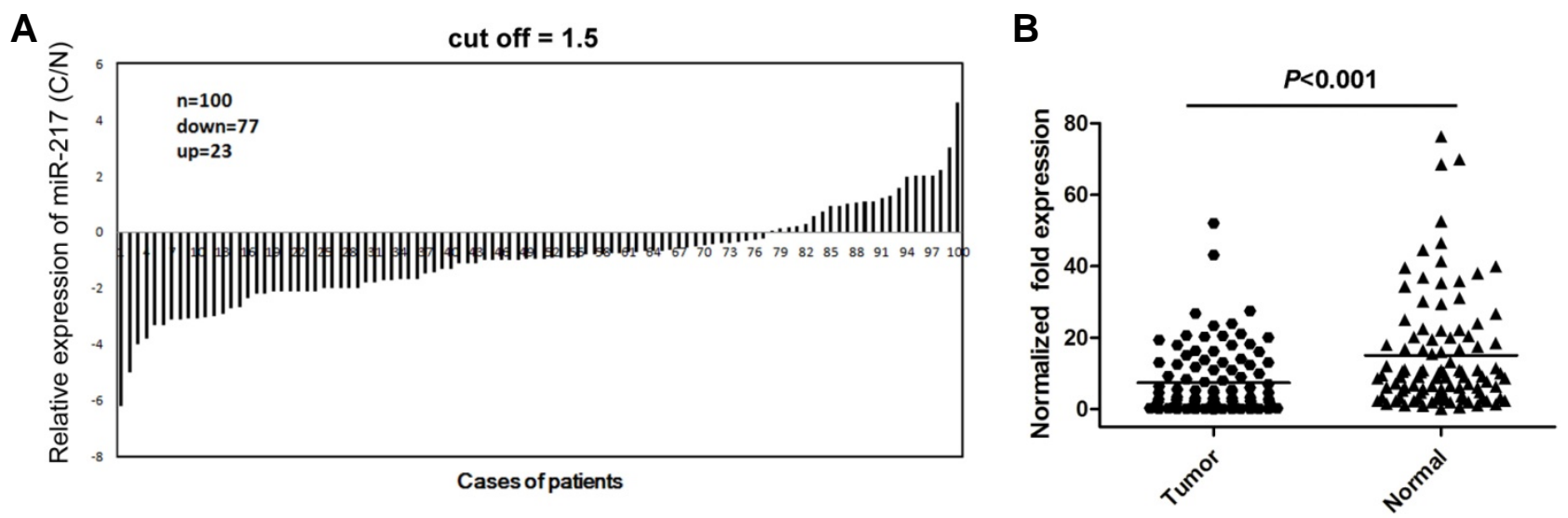

Fig. 1. MiR-217 expression in lung cancer clinical samples. (A) MiR-217 expression was detected by real-time PCR in 100 pairs of lung cancer tissues compared with their matched adjacent non-neoplastic tissues. A total of 77 samples showed down-regulated miR-217. (B) MiR-217 expression in the lung cancer tissues was significantly lower than that in adjacent tissues.

A

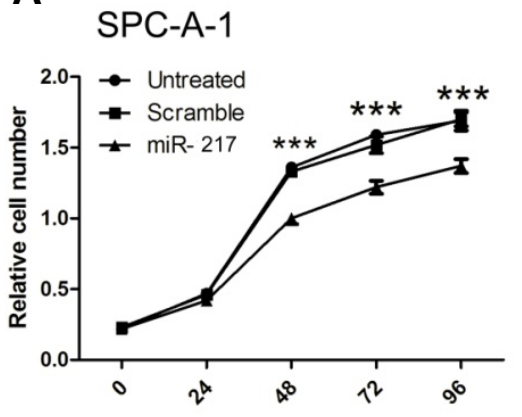

B

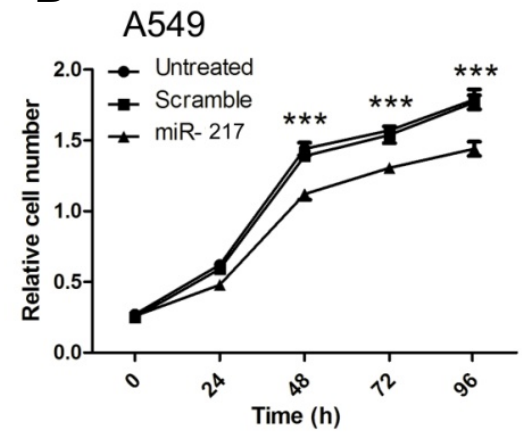

C

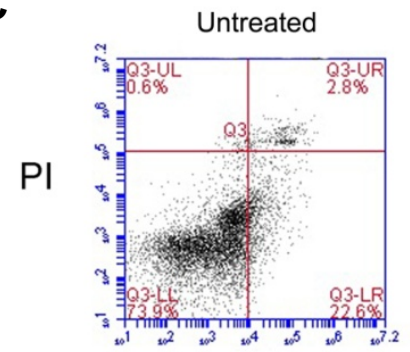

Annexin V

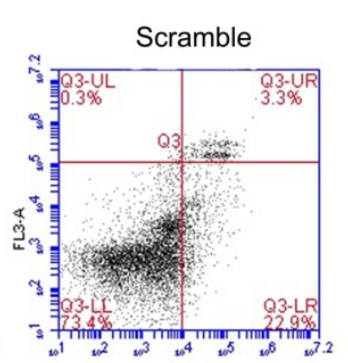

Annexin V

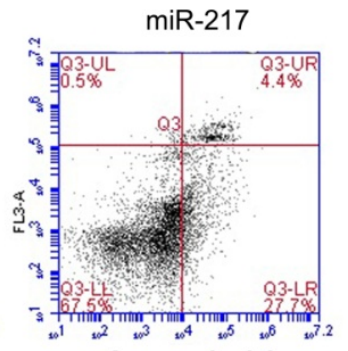

Annexin V

SPC-A-1

D

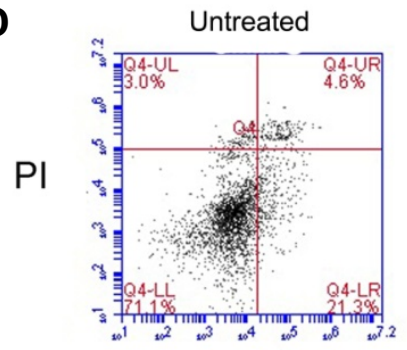

Annexin V

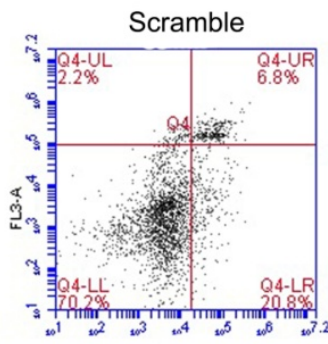

Annexin V

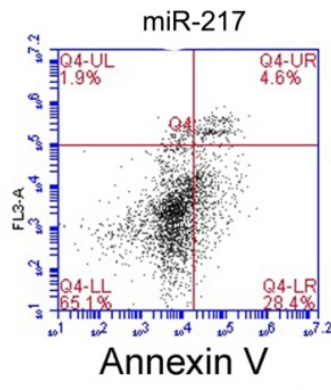

Fig. 2. MiR-217 overexpression regulates lung cancer cell growth. (A, B) MiR-217 overexpression inhibits lung cancer cell proliferation in SPC-A-1 and A549. The growth index was assessed after 0, 1, 2, 3, and 4 days. The bars represent the mean \pm SD of three independent experiments $\left({ }^{* * *} p<0.001\right)$. (C, D) MiR-217 overexpression promotes lung cancer cell apoptosis in SPC-A-1 and A549. The percentage of early apoptotic cells in the scramble group was approximately $22.9 \%$ in SPC-A1 and $20.8 \%$ in A549. However, these percentages were increased to $27.7 \%$ in SPC-A-1 and $28.4 \%$ in A549 after miR217 transfection. 
A

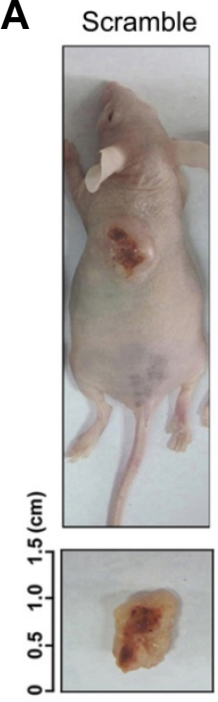

Vector-miR-217

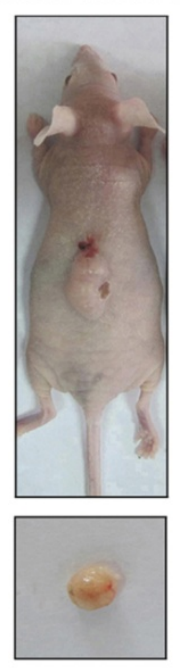

D

Scramble Vector-miR-217

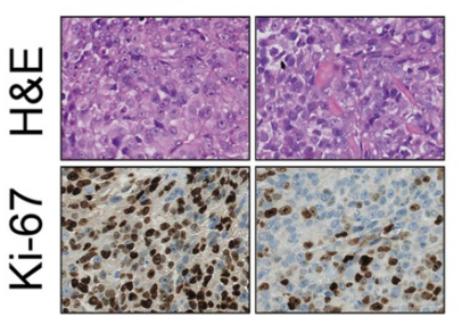

B
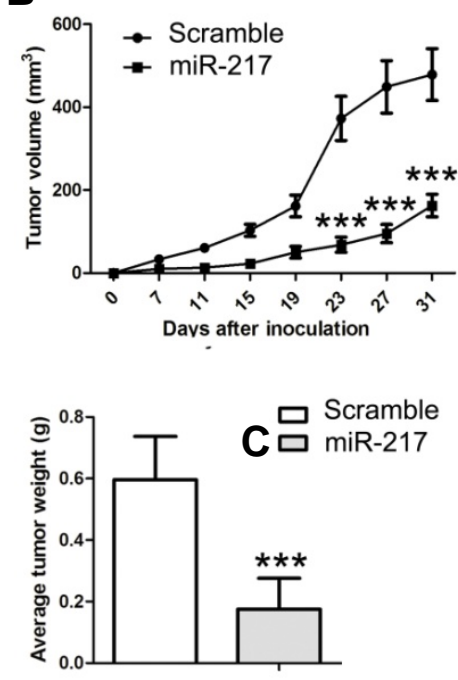

$\mathbf{F}$

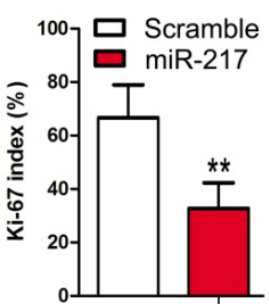

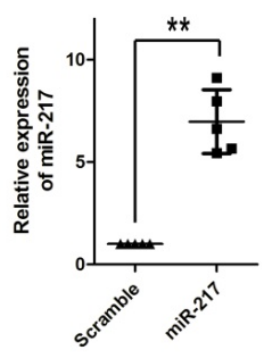

Fig. 3. miR-217 overexpression suppressestumour growth in vivo. (A) Scramble- and miR-217-transfected A549 cells were injected s.c. into the posterior flank of nude mice. The image represents tumour growth at 6 weeks after inoculation. $(B, C)$ Tumour volume and weight were calculated. All data are shown as mean \pm SD. Both tumour volume and weight were significantly reduced by miR-217 overexpression. ( ${ }^{* \star} p$ $<0.001$ ). (D) HE staining demonstrated tumour formation. Ki-67 expression was measured by immunohistochemistry from tumours to evaluate the proliferation ability of tumour cells. (E) Ki-67 index was significantly lower in the miR-217-overexpressing cells than in the control, indicating a weak proliferation ability $\left({ }^{* *} p<0.01\right)$. (F) MiR-217 expression in tumour. MiR-217 expression in the miR-217 overexpressing cells was significantly higher than that in the control. tion for $2 \mathrm{~h}$ at room temperature, anti-Bad/p/Bad antibody (Bioworld, USA) at 1:500 dilution, anti-AKT/p-AKT antibody (CST, USA) at 1:1000 dilution and anti-GAPDH antibody (Proteintech, USA) at 1:30,000 dilution. After washing twice with TBST, the membranes were incubated with goat anti-rabbit antibody (zsgbbio, China) at 1:5000 and 1:50000 dilutions for $2 \mathrm{~h}$.

\section{Statistics}

Each of the experiments was at least performed triplicate. Student's $t$-test (two-tailed) and the $\mathrm{x}^{2}$ test were performed, and statistically significant level was set at $\alpha=0.05$ two-side). Mean $\pm S D$ is displayed in the figures.

\section{RESULTS}

Expression of miR-217 in clinical lung cancer patients The expression of miR-217 in 100 patients (56 males/44 females, mean age of 53 years) was detected by RT-PCR. The expression of miR-217 was down-regulated in $77(77 \%)$ of the 100 lung cancer samples compared with adjacent tissues when the cut off was set to 1.5 (Fig. 1A). Meanwhile, miR-217 was up-regulated in $23(23 \%)$ of the 100 lung cancer samples. In general, miR-217 expression was frequently down-regulated in lung cancer tissues. The difference in miR-217 expression was significant between lung cancer tissues and adjacent tissues ( $p=0.00079$, Fig. 1B).

\section{MiR-217 inhibits cell proliferation in vitro and in vivo}

The results of CCK8 growth assays at 0,1,2, 3, and 4 days after miR-217 and scramble transfection are shown in Figs. 2A and 2B. Compared with scramble transfection, miR-217 transfection significantly reduced the proliferation of A549 and A549 and SPC-A-1 cells. We investigated the effect of miR-217 on the apoptosis of A549 and SPC-A-1 cells after miR-217 transfection. Scramble transfection induced the early apoptosis of $22.9 \%$ SPC-A-1 cells and 20.8\% A549 cells; meanwhile, miR-217 transfection induced the early apoptosis of $27.7 \%$ SPC-A-1 cells and $28.4 \%$ A549 cells (Figs. 2C and 2D). These results indicate that miR-217 can suppress lung cancer cell survival by inducing early apoptosis.

To further verify these findings, an in vivo model was constructed. Scramble-transfected and miR-217-overexpressing A549 cells $\left(6 \times 10^{6}\right.$ cells) were inoculated s.c. into the dorsal flanks of 15 mice (five for each group). After 6 weeks, tumour growth was significantly slower in the miR-217-overexpressing mice than in the control mice (Figs. 3A and 3B). In agreement with the tumour growth curve, the weights of tumours induced by scramble transfection were significantly higher than those induced by miR-217 overexpression (Fig. 3C). Similarly, immunohistochemical analysis was performed to measure the protein levels of $\mathrm{Ki}-67$ in the tumour tissues. Lower Ki-67 index was obtained in the miR-217-transfected cell tissues than in the controls (Figs. 3D and 3E). This result indicates that miR-217 overexpression can limit the proliferation of lung cancer cells in vivo.

MiR-217 inhibits lung cancer cell migration and invasion We further studied the effects of miR-217 on cell migration and invasion using a wound healing/scratch assay in SPC-A-1 and A549 cells with or without miR-217 mimic transfection. Results showed that both SPC-A-1 and A549 cell lines with miR-217 overexpression showed less migration ability than the scramble 
A
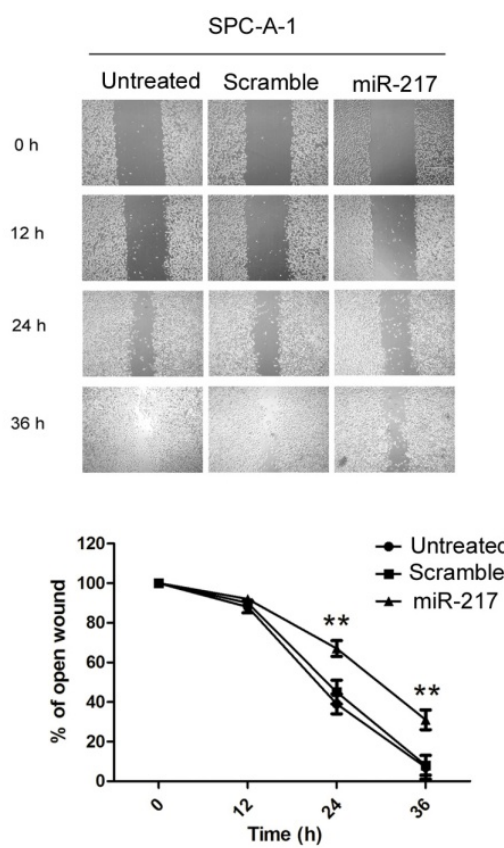

B
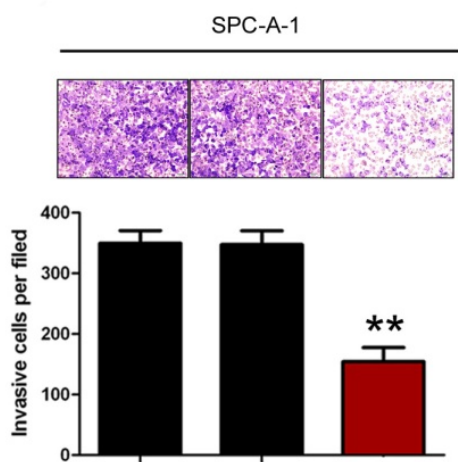

Untreated Scramble miR-217
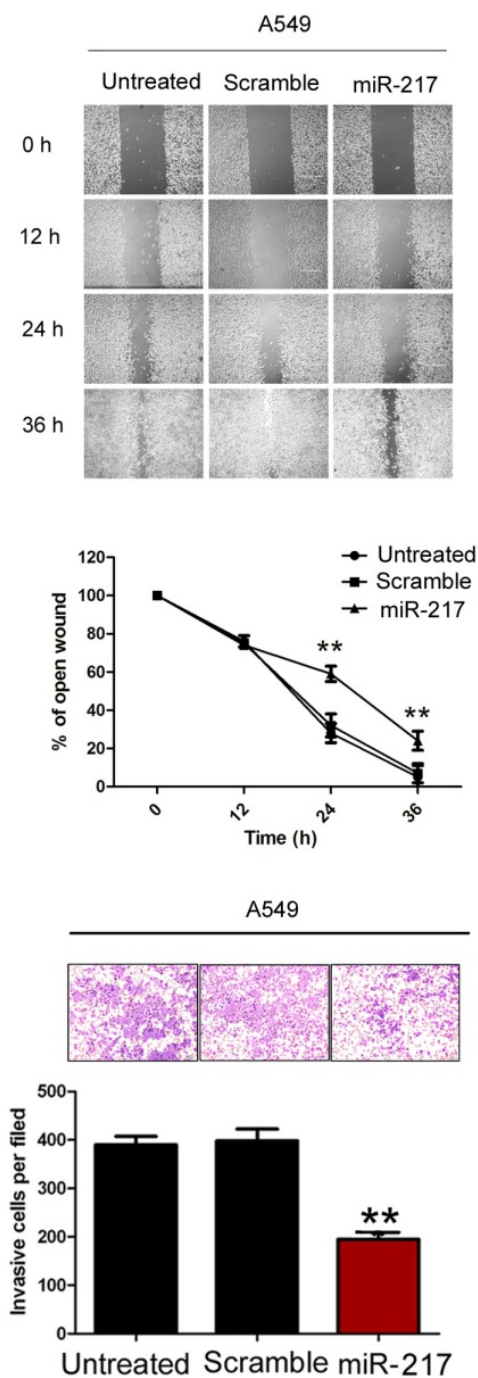

Fig. 4. MiR-217 overexpression inhibits lung cancer cell migration and invasion. (A) SPC-A-1 and A549 cells were not transfected or transfected with miR-217 mimics or scramble for $24 \mathrm{~h}$, and wounds were made. The relative ratio of wound closure per field is shown. The lung cancer cells transfected with miR-217 showed a significantly higher migration speed than the control. (B) SPC-A-1 and A549 cells were not transfected or transfected with miR-217 mimics or scramble for $24 \mathrm{~h}$, and transwell invasion assay was performed. The relative ratio of invasive cells per field is shown. The magnification for identification of migration is $\times 400$ and invasion is $\times 40\left({ }^{* *} p<0.01\right)$. The migration ability of lung cancer cells was inhibited by miR-217 overexpression. and untreated groups at 24 and $36 \mathrm{~h}$ after wound creation (Fig 4A).

We also detected the invasion abilities of lung cancer cells after miR-217 overexpression in SPC-A-1 and A549 cells. The cells with miR-217 overexpression showed significantly lower invasiveness than the scramble or untreated groups (Fig.4B). These results indicate that miR-217 regulates lung cancer cell migration and invasiveness. Therefore, the down-regulation of miR-217 may strengthen tumour metastasis in lung carcinogenesis.

\section{MiR-217 targets KRAS in lung cancer}

As predicted by Targetscan, complementarity can be found between has-miR-217 and KRAS 3'-UTR (Fig. 5A). To test whether or not KRAS is a target of miR-217, the plasmid pMIR-reportor containing the wild-type 3'-UTR region of KRAS downstream of the luciferase coding region (Fig. 5A, KRAS_WT) was constructed. HEK-293T cells were co-transfected with reporter plasmid (KRAS_WT) and scramble. Luciferase activity was markedly reduced by approximately $58 \%$ in the miR-217overexpressing cells. The same assay was performed for another reporter plasmid containing mutated KRAS 3'-UTR in miR-
217 binding sites. As expected, the miR-217-induced inhibition of luciferase activity was partly removed with binding site 1 orbinding site 2 mutant and almost abolished in the KRAS_MUT double mutant (Fig. 5B). This result suggests that the conserved region regulates the functions of miR-217.

We tested five other potential target genes using the same methods. The results of luciferase miRNA target reporter assay showed that the five potential target genes were not the target ofmiR-217 (Supplementary Fig. S1).

SPC-A-1 and A549 cells were transfected with miR-217 to further investigate the interaction between miR-217 and KRAS. Western blot was conducted to measure the level of KRAS protein. The protein expression of KRAS was down-regulated in the miR217-treated SPC-A-1 and A549 cells but not in the scramble or untreated cells (Fig. 5D). The mRNA expression of KRAS was determined by real-time PCR. No significant difference was observed between miR-217-treated and scramble-treated or untreated SPC-A-1 and A549 cells (Fig. 5C). These results suggestthat miR-217 directly recognises the $3^{\prime}$-UTR of KRAS mRNA and inhibits KRAS translation. Thus, miR-217 down-regulation in lung cancer inhibits KRAS suppression, which consequently decelerates tumourigenesis. Interestingly, the phosphorylation 
A

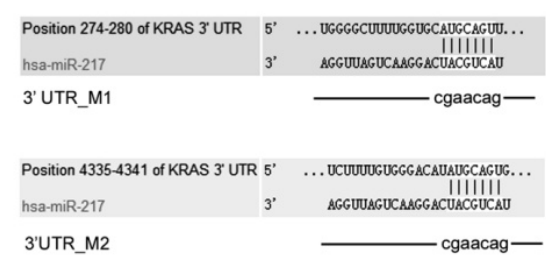

C

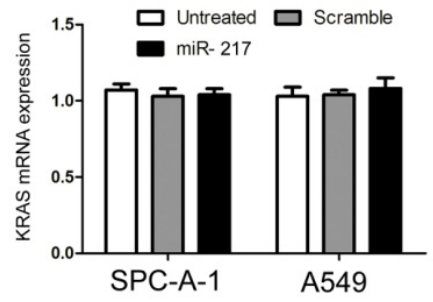

D

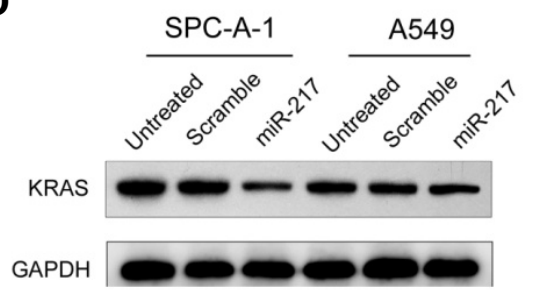

B

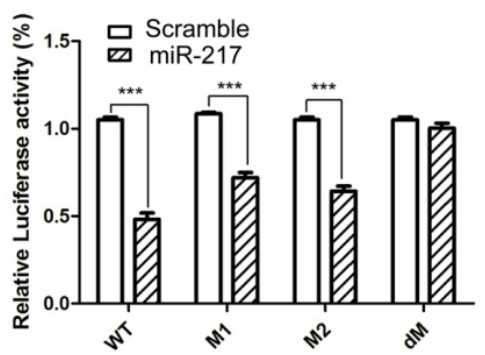

E

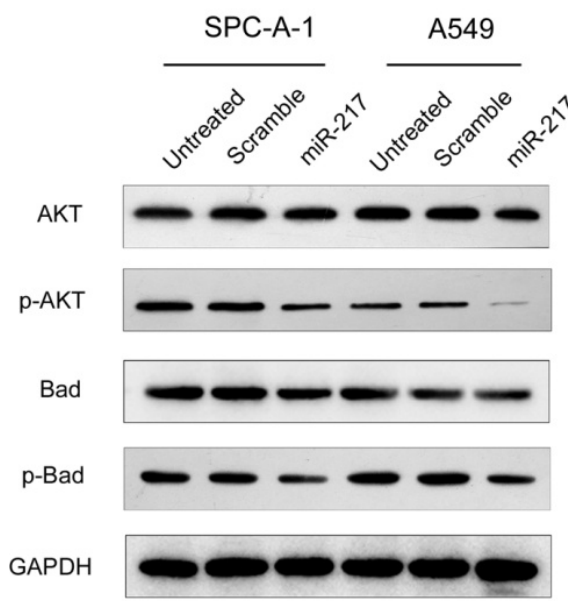

Fig. 5. KRAS is a direct target of miR217. (A) Sequence of the miR-217 binding sites within the human KRAS 3 '-UTR and a schematic of the reporter constructs showing the KRAS $3^{\prime}$-UTR sequence and the mutated KRAS 3 '-UTR sequence (M1, M2). (B) Luciferase activity of the KRAS_WT and KRAS_MUT reporters in the presence of $10 \mathrm{nmol} / \mathrm{L}$ of miR-217 mimic or scramble $\left({ }^{* * *} \mathrm{p}<\right.$ 0.001). (C) Relative expression of KRAS in SPC-A-1 and A549 cells not transfected or transfected with miR-217 mimics or scramble for $24 \mathrm{~h}$, indicating that the miR-217 did not inhibit kras mRNA expression. (D) Immunoblotting of KRAS in SPC-A-1 and A549 cells not transfected or transfected with miR-217 mimic or scramble, which showed that the miR217 reduced the KRAS protein level, indicating that KRAS can be a direct target of miR-217. All data are shown as mean $\pm S D$. (E) Immunoblotting of AKT, p-AKT, Bad and p-Bad in SPC-A-1 and A549. levels of Akt and Bad were similarly altered to the expression level of KRAS (Fig. 5E).

\section{Up-regulation of miR-217 enhances the sensitivity of SPC-A-1 and A549 cells to cisplatin in vitro}

Previous research demonstrated that miRNAdysregulation is related to the chemoresistance of cancers, including lung cancer (Bian et al., 2011; Giovannetti et al., 2010). However, to the best of our knowledge, the relationship between miR-217 expression and lung cancer sensitivity has yet to be explored. Therefore, we treated scramble- or miR-217-transfected SPC-A-1 and A549 cells with $0,5,10,15,20$ and $25 \mu \mathrm{g} / \mathrm{ml}$ of cisplatin for $12 \mathrm{~h}$. The results of CCK8 assay indicated that miR-217 up-regulation significantly decreased the cell viability of SPC-A-1 and A549 cells in response to cisplatin in a dose-dependent manner (Figs. $6 \mathrm{~A}$ and $6 \mathrm{C}$ ). Moreover, cisplatin up-regulated miR-217 in the two cell lines. The expression level of miR-217 increased with cisplatin in a dose-and time-dependent manner (Figs. 6B and 6D). To confirm this effect, we evaluated the mRNA and proteinexpression levels of KRAS. As expected, the mRNA and protein levels of KRAS significantly reduced, coinciding with miR-217 expression (Figs. 6E and 6F). We silenced miR-217 using antagomiR to investigate the effect of miR-217 on the sensitivity oflung cancer cell lines SPC-A-1 and A549 to cisplastin. Compared with miR-217 mimic, antagomiR-217 caused cisplatin torrent in both SPC-A-1 and A549 cells (Figs. 6G and 6H). This result demonstrates that miR-217 can enhance the sensitivity of SPC-A-1 and A549 cells to cisplatin.

\section{DISCUSSION}

MiRNAs as regulators play important roles in tumourigenesis (Deng et al., 2008; Pasquinelli, 2012). MiRNAs exhibit differential expression modes in cancers and function as tumour suppressor genes or oncogenes by targeting specific target genes (Zhang, 2007). Generally, miRNAs that function as tumour suppressors are expressed at relatively low levels, whereas oncogenic miRNAs exhibit relatively high expression levels in tumours. Therefore, miRNAs may serve as new biomarkers to predict clinical outcomes in the future (Esteller, 2011).

MiR-217 is a special miRNA that can function as an oncogene or a tumour suppressor gene depending on cell type. For example, miR-217 targets the oncogene SirT1 or KRAS in endothelial cells and pancreatic ductal adenocarcinoma cells (Menghini et al., 2009; Zhao et al., 2010) but targets the tumour suppressor gene PTEN in kidney cells (Kato et al., 2009).

Cisplatin is a commonly used chemotherapeutic drug. MiRNAdysregulation can alter cisplatinchemoresistance in cancer cells (Sorrentino et al., 2008; Yu et al., 2010). MicroRNA-451 can increase the cisplatin sensitivity of lung cancer cells (Bian et al., 2011).

The present study provided insights into the association of miR-217 expression with cisplatin cell resistance in lung cancer. We evaluated miR-217 expression in lung cancer patients. Results showed that $77 \%$ of lung cancer clinical samples showed significantly lower miR-217 expression than adjacent normal tissues $(p=0.00079$ ). This result suggests that miR-217 may function as a tumour suppressor. In vitro and in vivo studies proved that miR-217 can also serve as a tumour suppressor gene in human lung cancer progression. The results of the as 
A

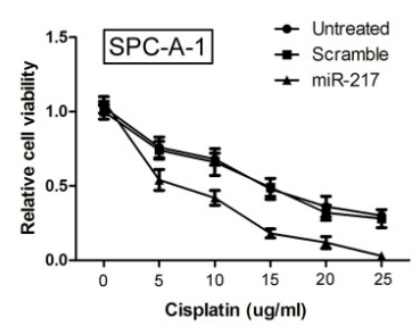

C

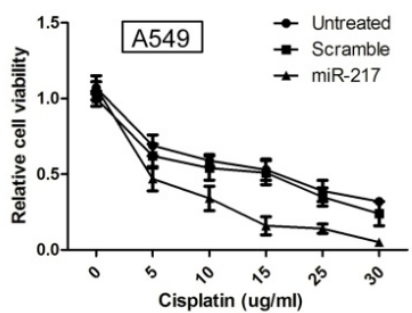

$\mathbf{E}$

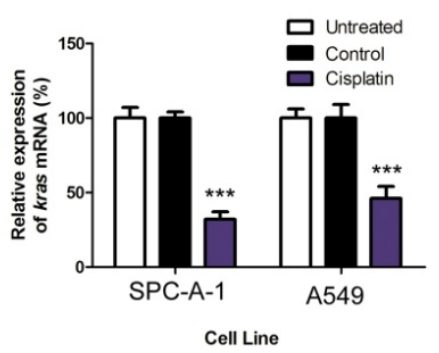

B

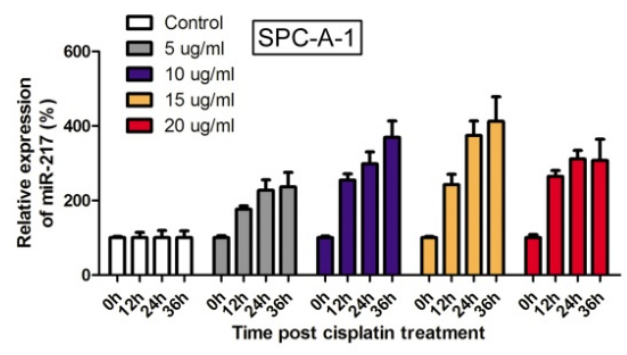

D

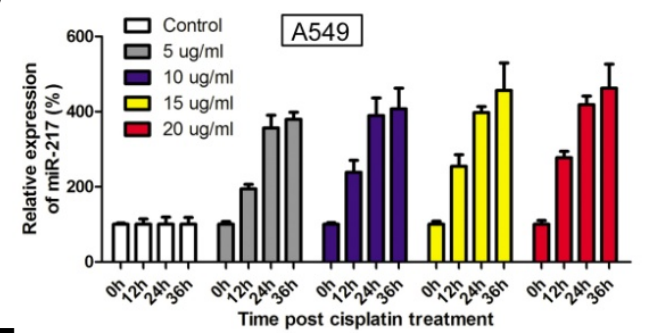

$\mathbf{F}$

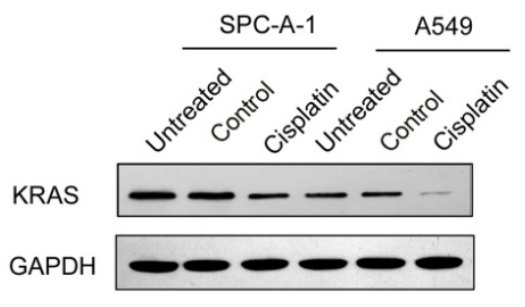

H
Fig. 6. Effect of miR-217 upregulation on the sensitivity of SPCA-1 and A549 cells to cisplatin. (A, C) Effects of various concentrations of cisplatin on SPC-A-1 and A549 cells for $12 \mathrm{~h}$ as assessed by CCK8 assay, indicating that miR-217 upregulation can significantly decrease the cell viability of SPC-A-1 and A549 cells in response to cisplatin in a dose-dependent manner. $(B, D)$ Effects of various concentrations and various time of cisplatin treatment on miR-217 expression in SPC-A-1 and A549 cells. MiR-217 was up-regulated by the cisplatin treatment in the two cell lines. (E, F) Effects of cisplatin treatment on KRAS mRNA and protein expression in SPC-A-1 and A549 cells. The mRNA and protein levels of KRAS were down-regulated by cisplatin treatment. (G, H) miR-217 was silenced using antagomiR to investigate the effect of miR-217 on the sensitivity of lung cancer cells. AntagomiR-217 treatment caused cisplatin accumulation in both SPCA-1 and A549 cells. Scramble, negative control of microRNA mimic miR217 or antagomiR. Control, negative control of cisplatin.
G

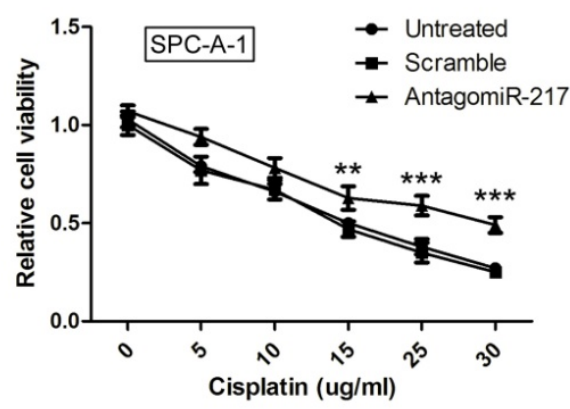

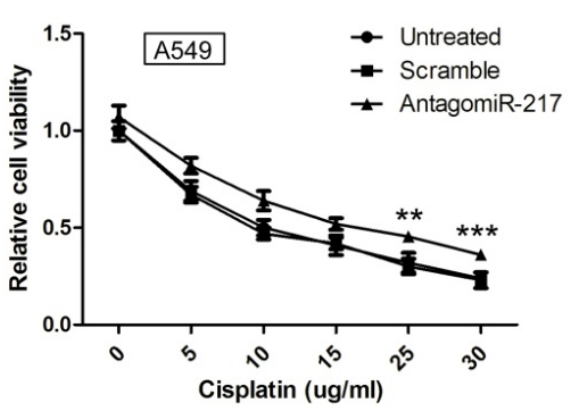

says on cell proliferation, apoptosis, migration and invasion may be used to further understand the mechanism by which miR-217 contributes to lung cancer tumourigenesis and progression. KRAS is a direct target of miR-217 in pancreatic cancer (Zhao et al., 2010). In the present study, miR-217 overexpression decreased KRAS levels in two lung cancer cell lines. This result indicates that KRAS also serves as a target of miR-217 in lung cancer. The PI3K/Akt pathway is a major downstream effector of KRAS signalling. Downstream factors such as ERK, Bad, Bcl-xl and NF-kB have been linked to the Akt pathway. AKT is a crucial factor of the PI3K/AKT pathway. The Akt-mediated hyperphosphorylation of Bad may promote cell survival in cancer cells. The results of Western blot affirmed our speculation that decreased Bad phosphorylation increases apoptosis in pre-miR-217transfected cells. Previous research showed that two other pro- teins SIRT1 and E2F3 may also be targets of miR-217 (Su et al., 2014; Zhang et al., 2012). However, we failed to verify this finding in lung cancer.

This study is the first to report that miR-217 overexpression can reduce cisplatin cell resistance in lung cancer cell lines. Therefore, combining cisplatin with miR-217 regulation may serve as a potential approach for lung cancer therapy. High cisplatin doses can cause toxic effects, such as nephrotoxicity and ototoxicity. The results of this study showed that the sensitivity of lung cancer cells to cisplatin significantly increased. Therefore, we speculate that using cisplatin in combination with miR-217 as a potential approach for lung cancer therapy may achieve lowdose cisplatin application and reduce cisplatin toxic effects.

No significant changes in KRAS mRNA level were observed (Fig. 5C). This result indicates that miR-217 may function as a 
post-transcriptional regulation factor of KRAS. However, the cisplatin-induced up-regulation of miR-217 significantly changed the mRNA level of KRAS. This result indicates that cisplatin targets other genes aside from miR-217 and influences KRAS mRNA. These data reflect the complexity of biological control. However, we failed to find the key regulator in this process. Thus, this phenomenon warrants further investigations.

In conclusion, miR-217 is frequently down-regulated in lung cancer and acts as a tumour suppressor in lung cancer cells by targeting KRAS. MiR-217 up-regulation enhances the sensitivity of SPC-A-1 and A549 cells to cisplatin. These results provided insights into the functions of miR-217 in cisplatinchemoresistance and the rational development of new targeted combinations against lung cancer.

Note: Supplementary information is available on the Molecules and Cells website (www.molcells.org).

\section{ACKNOWLEDGMENTS}

This study was supported by grants from the Henan Province's Technology Key Project of Medical Science and Technology (2011030004). We declare no conflict of interest.

\section{REFERENCES}

Ambs, S., Prueitt, R.L., Yi, M., Hudson, R.S., Howe, T.M., Petrocca, F., Wallace, T.A., Liu, C.G., Volinia, S., Calin, G.A., et al. (2008). Genomic profiling of microRNA and messenger RNA reveals deregulated microRNA expression in prostate cancer. Cancer Res. 68, 6162-6170.

Bian, H.B., Pan, X., Yang, J.S., Wang, Z.X., and De, W. (2011). Upregulation of microRNA-451 increases cisplatin sensitivity of non-small cell lung cancer cell line (A549). J. Exp. Clin. Cancer Res. 30, 20.

Cimmino, A., Calin, G.A., Fabbri, M., Iorio, M.V., Ferracin, M., Shimizu, M., Wojcik, S.E., Aqeilan, R.I., Zupo, S., Dono, M., et al. (2005). miR-15 and miR-16 induce apoptosis by targeting BCL2. Proc. Natl. Acad. Sci. USA 102, 13944-13949.

Deng, S., Calin, G.A., Croce, C.M., Coukos, G., and Zhang, L. (2008). Mechanisms of microRNA deregulation in human cancer. Cell Cycle 7, 2643-2646.

Engels, B.M., and Hutvagner, G. (2006). Principles and effects of microRNA-mediated post-transcriptional gene regulation. Oncogene 25, 6163-6169.

Esteller, M. (2011). Non-coding RNAs in human disease. Nat. Rev. Genet. 12, 861-874.

Garzon, R., Calin, G.A., and Croce, C.M. (2009). MicroRNAs in cancer. Annu. Rev. Med. 60, 167-179.

Giovannetti, E., Funel, N., Peters, G.J., Del Chiaro, M., Erozenci, L.A., Vasile, E., Leon, L.G., Pollina, L.E., Groen, A., Falcone, A., et al. (2010). MicroRNA-21 in pancreatic cancer: correlation with clinical outcome and pharmacologic aspects underlying its role in the modulation of gemcitabine activity. Cancer Res. 70, 45284538.

Guttilla, I.K., and White, B.A. (2009). Coordinate regulation of FOXO1 by miR-27a, miR-96, and miR-182 in breast cancer cells. J. Biol. Chem. 284, 23204-23216.

Inomata, M., Tagawa, H., Guo, Y.M., Kameoka, Y., Takahashi, N., and Sawada, K. (2009). MicroRNA-17-92 down-regulates expression of distinct targets in different B-cell lymphoma subtypes. Blood 113, 396-402.

Iorio, M.V., Visone, R., Di Leva, G., Donati, V., Petrocca, F., Casalini, P., Taccioli, C., Volinia, S., Liu, C.G., Alder, H., et al. (2007). Mi
croRNA signatures in human ovarian cancer. Cancer Res. 67 8699-8707.

Jemal, A., Siegel, R., Xu, J., and Ward, E. (2010). Cancer statistics, 2010. CA Cancer J. Clin. 60, 277-300.

Johnson, S.M., Grosshans, H., Shingara, J., Byrom, M., Jarvis, R., Cheng, A., Labourier, E., Reinert, K.L., Brown, D., and Slack, F.J. (2005). RAS is regulated by the let-7 microRNA family. Cell 120, 635-647.

Kato, M., Putta, S., Wang, M., Yuan, H., Lanting, L., Nair, I., Gunn, A. Nakagawa, Y., Shimano, H., Todorov, I., et al. (2009). TGF-beta activates Akt kinase through a microRNA-dependent amplifying circuit targeting PTEN. Nat. Cell Biol. 11, 881-889.

Ma, J., Dong, C., and Ji, C. (2010). MicroRNA and drug resistance. Cancer Gene Ther. 17, 523-531.

Menghini, R., Casagrande, V., Cardellini, M., Martelli, E., Terrinoni, A. Amati, F., Vasa-Nicotera, M., Ippoliti, A., Novelli, G., Melino, G., et al. (2009). MicroRNA 217 modulates endothelial cell senescence via silent information regulator 1 . Circulation 120, 1524-1532.

Pasquinelli, A.E. (2012). MicroRNAs and their targets: recognition, regulation and an emerging reciprocal relationship. Nat. Rev. Genet. 13, 271-282.

Qi, L., Bart, J., Tan, L.P., Platteel, I., Sluis, T., Huitema, S., Harms, G. $\mathrm{Fu}$, L., Hollema, H., and Berg, A. (2009). Expression of miR-21 and its targets (PTEN, PDCD4, TM1) in flat epithelial atypia of the breast in relation to ductal carcinoma in situ and invasive carcinoma. BMC Cancer 9, 163.

Schepeler, T., Reinert, J.T., Ostenfeld, M.S., Christensen, L.L., Silahtaroglu, A.N., Dyrskjot, L., Wiuf, C., Sorensen, F.J., Kruhoffer, M., Laurberg, S., et al. (2008). Diagnostic and prognostic microRNAs in stage II colon cancer. Cancer Res. 68, 6416-6424.

Slack, F.J., and Weidhaas, J.B. (2008). MicroRNA in cancer prognosis. N Engl. J. Med. 359, 2720-2722.

Sorrentino, A., Liu, C.G., Addario, A., Peschle, C., Scambia, G., and Ferlini, C. (2008). Role of microRNAs in drug-resistant ovarian cancer cells. Gynecol. Oncol. 111, 478-486.

Su, J., Wang, Q., Liu, Y., and Zhong, M. (2014). miR-217 inhibits invasion of hepatocellular carcinoma cells through direct suppression of E2F3. Mol. Cell. Biochem.392, 289-296.

Tomari, Y., and Zamore, P.D. (2005). Perspective: machines for RNAi. Genes Dev. 19, 517-529.

Wang, R., Wang, Z.X., Yang, J.S., Pan, X., De, W., and Chen, L.B. (2011). MicroRNA-451 functions as a tumor suppressor in human non-small cell lung cancer by targeting ras-related protein 14 (RAB14). Oncogene 30, 2644-2658.

Yu, S., Lu, Z., Liu, C., Meng, Y., Ma, Y., Zhao, W., Liu, J., Yu, J., and Chen, J. (2010a). miRNA-96 suppresses KRAS and functions as a tumor suppressor gene in pancreatic cancer. Cancer Res. 70, 6015-6025.

Yu, Z.W., Zhong, L.P., Ji, T., Zhang, P., Chen, W.T., and Zhang, C.P. (2010b). MicroRNAs contribute to the chemoresistance of cisplatin in tongue squamous cell carcinoma lines. Oral Oncol. 46, 317-322.

Zhang, B., Pan, X., Cobb, G.P., and Anderson, T.A. (2007). microRNAs as oncogenes and tumor suppressors. Dev. Biol.302, 1-12.

Zhang, T., Wang, Q., Zhao, D., Cui, Y., Cao, B., Guo, L., and Lu, S.H. (2011). The oncogenetic role of microRNA-31 as a potential biomarker in oesophageal squamous cell carcinoma. Clin. Sci. 121, 437-447.

Zhang, H.S., Wu, T.C., Sang, W.W., and Ruan, Z. (2012). MiR-217 is involved in Tat-induced HIV-1 long terminal repeat (LTR) transactivation by down-regulation of SIRT1. Biochim. Biophys. Acta 1823, 1017-1023.

Zhao, W.G., Yu, S.N., Lu, Z.H., Ma, Y.H., Gu, Y.M., and Chen, J. (2010). The miR-217 microRNA functions as a potential tumor suppressor in pancreatic ductal adenocarcinoma by targeting KRAS. Carcinogenesis 31, 1726-1733. 